\title{
Inhibition of pulmonary fibrosis in mice by CXCL10 requires glycosaminoglycan binding and syndecan-4
}

\author{
Dianhua Jiang, ${ }^{1}$ Jiurong Liang, ${ }^{1}$ Gabriele S. Campanella, ${ }^{2}$ Rishu Guo, ${ }^{1}$ Shuang Yu, ${ }^{3}$ Ting Xie, ${ }^{1,4}$ \\ Ningshan Liu, ${ }^{1}$ Yoosun Jung, ${ }^{1}$ Robert Homer, ${ }^{5}$ Eric B. Meltzer, ${ }^{1}$ Yuejuan Li, ${ }^{1}$ Andrew M. Tager, ${ }^{2}$ \\ Paul F. Goetinck, ${ }^{6}$ Andrew D. Luster, ${ }^{2}$ and Paul W. Noble ${ }^{1}$

\begin{abstract}
'Division of Pulmonary, Allergy, and Critical Care Medicine, Department of Medicine, Duke University School of Medicine, Durham, North Carolina, USA. ${ }^{2}$ Center for Immunology and Inflammatory Diseases, Division of Rheumatology, Allergy and Immunology, Massachusetts General Hospital, Harvard Medical School, Charlestown, Massachusetts, USA. ${ }^{3}$ Department of Medicine, Yale University School of Medicine, New Haven, Connecticut, USA ${ }^{4}$ Beijing University of Chinese Medicine, Beijing, China. ${ }^{5}$ Department of Pathology, Yale University School of Medicine, New Haven, Connecticut, USA.
\end{abstract} \\ ${ }^{6}$ Cutaneous Biology Research Center, Massachusetts General Hospital, Harvard Medical School, Charlestown, Massachusetts, USA.
}

\begin{abstract}
Pulmonary fibrosis is a progressive, dysregulated response to injury culminating in compromised lung function due to excess extracellular matrix production. The heparan sulfate proteoglycan syndecan-4 is important in mediating fibroblast-matrix interactions, but its role in pulmonary fibrosis has not been explored. To investigate this issue, we used intratracheal instillation of bleomycin as a model of acute lung injury and fibrosis. We found that bleomycin treatment increased syndecan-4 expression. Moreover, we observed a marked decrease in neutrophil recruitment and an increase in both myofibroblast recruitment and interstitial fibrosis in bleomycin-treated syndecan-4-null $\left(S d c 4^{-/-}\right)$mice. Subsequently, we identified a direct interaction between CXCL10, an antifibrotic chemokine, and syndecan-4 that inhibited primary lung fibroblast migration during fibrosis; mutation of the heparin-binding domain, but not the CXCR3 domain, of CXCL10 diminished this effect. Similarly, migration of fibroblasts from patients with pulmonary fibrosis was inhibited in the presence of CXCL10 protein defective in CXCR3 binding. Furthermore, administration of recombinant CXCL10 protein inhibited fibrosis in WT mice, but not in $S d c 4^{-/-}$mice. Collectively, these data suggest that the direct interaction of syndecan-4 and CXCL10 in the lung interstitial compartment serves to inhibit fibroblast recruitment and subsequent fibrosis. Thus, administration of CXCL10 protein defective in CXCR3 binding may represent a novel therapy for pulmonary fibrosis.
\end{abstract}

\section{Introduction}

The molecular mechanisms of pulmonary fibrosis remain poorly understood. When the normal host encounters an exogenous insult, endogenous pathways are activated to limit the extent of tissue injury and initiate repair processes. The tissue injury and subsequent repair responses must be tightly regulated. However, in susceptible hosts, a limited injury will evoke an exaggerated repair response that causes extensive fibrogenesis and impaired end organ function. The latter is a dysregulated repair process (1) and may represent abnormalities in host defense (2). Although considerable information is known about the host response to infectious insults, the mechanisms that control inflammation and fibrosis in response to noninfectious or sterile injury are less well studied.

The essential hallmark of severe pulmonary fibrosis is exorbitant production of extracellular matrix molecules, including collagen, fibronectin, tenascin, and proteoglycans. Alveolar type II cell injury and apoptosis may be important early features in the pathogenesis of pulmonary fibrosis (3). Fibroblasts from patients with pulmonary fibrosis have a unique phenotype, different growth rates (4), and altered production of tissue inhibitors of metalloproteinases and other mediators (5). Fibroblast migration is a critical feature of progressive tissue fibrosis (6) and is a dynamic process that is tightly regulated by growth factors such

Conflict of interest: The authors have declared that no conflict of interest exists. Citation for this article: JClin Invest. 2010;120(6):2049-2057. doi:10.1172/JCI38644 as FGF, TGF- $\beta 1$, and PDGF (7). However, the endogenous mechanisms that regulate the accumulation of fibroblasts in the lung interstitium remain poorly understood.

During lung injury and repair, chemokines, cytokines, and other mediators are released from various cell types, including epithelial cells, endothelial cells, fibroblasts, and leukocytes. Chemokines and chemokine receptors have been shown to play important roles in regulating injury and repair. In particular, the balance between CXC chemokines positive and negative for the ELR motif (i.e., amino acids Glu-Leu-Arg) has been shown to regulate progressive lung fibrosis (8). Exogenous CXCL10 attenuated bleomycin-induced pulmonary fibrosis (9) and inhibited fibroblast migration in response to alveolar lavage fluid from injured lung tissue (10). In addition, $\mathrm{Cxcl10}^{-/-}$mice exhibited increased pulmonary fibrosis in the same model (10). Furthermore, genetically targeting the CXCL10 receptor CXCR3 resulted in increased mortality with progressive interstitial fibrosis, demonstrating an essential role for CXCR3 in limiting tissue fibroproliferation (11). Although CXCL10 inhibited fibroblast migration (10), the mechanism remained uncertain, since lung fibroblasts do not express CXCR3 on the cell surface.

Syndecan-4 (encoded by $S d c 4$ ), the most ubiquitous of the 4 syndecan family members, is expressed in most tissues and cell types, although it is usually expressed at basal levels (12). It is a transmembrane heparan sulfate proteoglycan that acts cooperatively with integrins in generating signals necessary for the assembly of actin stress fibers and focal adhesions in a Rho-dependent manner 

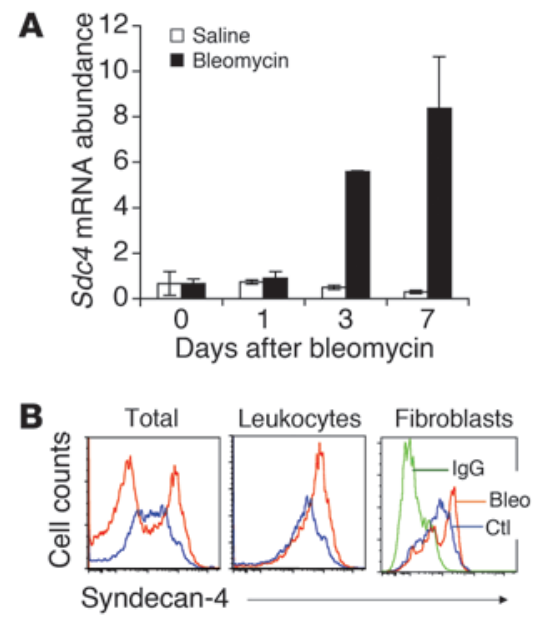

Figure 1

Noninfectious lung injury induced syndecan-4 expression. (A) Sdc4 mRNA was upregulated by bleomycin-induced lung injury. Bleomycin or saline was given to C57BL/6 mice intratracheally, and lungs were harvested 1,3 , and 7 days after injury. The mRNA levels in lung tissues were assessed by RT-PCR $(n=3-4)$. (B) Syndecan-4 cell surface expression was upregulated in lung tissues 7 days after bleomycin-induced injury. Bleomycin was given to C57BL/6 mice intratracheally, lungs were harvested 7 days after injury, and single cells were isolated by Dispase II and DNase I digestions. Cell surface expression of syndecan-4 was determined by staining cells with specific antibody to syndecan-4 ( $n=6$ [untreated]; 9 [bleomycin]). Flow plots showed syndecan-4 expression gated on total live cells and on leukocytes $\left(\mathrm{CD} 45^{+}\right)$. Fibroblasts isolated from lung tissue of mice untreated (Ctl) or treated with bleomycin (Bleo) for 7 days were also stained for syndecan-4. Negative staining with control IgG is shown in green.

(13). Through its heparan sulfate side chains, syndecan-4 interacts with an array of extracellular proteins, including growth factors such as basic FGF-2 (14) and TGF- $\beta$ (15), chemokines (16), adhesion molecules (13), and other extracellular matrix proteins (12). Engagement of syndecan-4 is essential for focal adhesion formation via certain integrins and for cell proliferation and migration in response to growth factors (17). The interactions may also facilitate growth factor signaling (14). Recent gene targeting studies suggest syndecan-4 plays a critical role in protecting against kidney injury (18), limiting endotoxin shock (19), and promoting wound healing (20). For example, a significant increase in susceptibility to

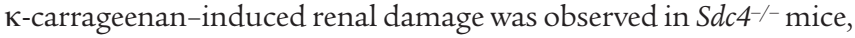
although renal organogenesis and function were normal in the unchallenged mice (18). Sdc4 $4^{--}$mice were also previously shown to be more susceptible to endotoxic shock (19).

However, the role of syndecan-4 in pulmonary fibrosis has not been investigated, although a few studies have explored the role of syndecan-4 in lung biology. For example, syndecan-4 expression was associated with the airway epithelial-mesenchymal trophic unit after exposure to ozone (21). Polyarginine treatment induced clustering of syndecan- 4 and syndecan- 1 as well as actin stress fiber formation in the lung (22). In the present study, we examined the role of syndecan-4 in noninfectious lung injury using intratracheal instillation of bleomycin as a model of acute lung injury $(11,23$, 24). We demonstrated that through its direct interaction with the chemokine CXCL10, syndecan-4 regulated lung inflammation and fibrosis by controlling fibroblast trafficking.

\section{Results}

Lung injury induces syndecan-4 expression. In order to elucidate the role of syndecan- 4 in lung inflammation and fibrosis, syndecan-4 expression was examined over time after bleomycin-induced lung injury in C57BL/6 mice. After treatment with bleomycin, we observed a marked increase in Sdc4 mRNA expression in whole lung tissue (Figure 1A). Expression of cell surface syndecan-4 was assessed with flow cytometric analysis, and increased syndecan-4 expression was detected on the cell surface of lung single-cell homogenates from bleomycin-injured lung compared with cells from control untreated mice (Figure 1B). The cell surface expression of syndecan-4 was significantly increased on leukocytes $\left(\mathrm{CD} 45^{+}\right)$and marginally increased on purified fibroblasts (Figure $1 \mathrm{~B}$ ). These data suggested that expression of syndecan-4 is upregulated in response to lung injury and that syndecan- 4 may play a role during tissue injury and repair.

$S d c 4^{-/-}$mice have an exaggerated fibrotic phenotype after noninfectious acute lung injury. To investigate the biological significance of the inducible expression of syndecan-4, we examined the inflammatory and fibrotic responses to lung injury in $S d c 4^{-/-}$mice. Recent studies with $S d c 4^{-/-}$mice suggested that syndecan-4 plays a nonredundant role in tissue injury (18) and repair (20). We observed a marked increase in lung fibrosis in $S d c 4^{-/-}$mice compared with littermate controls, as demonstrated by increased hydroxyproline content, elevated collagen staining by Trichrome, and increased immunofluorescence staining specific for collagen I (Figure 2, A-C). Fibroblasts with an activated myofibroblast phenotype have been described in the fibrotic foci that characterize progressive pulmonary fibrosis (4). Fibroblasts, especially myofibroblasts, are major effector cell types that produce extracellular matrix and provide the contractile forces during fibrogenesis (25). When we stained lung sections with anti- $\alpha$-SMA antibodies, a marker for newly appearing myofibroblasts, $S \mathrm{Cc}^{-/-}$mice showed a marked increase in cells staining positive for $\alpha$-SMA (Figure 2B). These data suggested that syndecan-4 plays a role in limiting fibrogenesis after noninfectious lung injury, possibly by restraining fibroblast/myofibroblast migration to areas of tissue injury.

Sdc4 $4^{-/}$mice show dysregulated inflammation after lung injury. We then investigated the role of syndecan- 4 in the inflammatory response to noninfectious acute lung injury. We observed a marked increase in total inflammatory cells in the BAL fluid in $\mathrm{Sdc4^{-/ }}$ mice compared with their WT littermates (Figure 3A). This may be a generalized phenotype for syndecan-4 deficiency, since we observed an increase in peritoneal cell inflammatory cell infiltration after thioglycollate stimulation (Supplemental Figure 1; supplemental material available online with this article; doi:10.1172/JCI38644DS1). The differential cell counts of BAL cells revealed that the total increase in inflammatory cells was mostly due to macrophages and lymphocytes (Figure 3, B and E). Furthermore, we identified a relative deficiency in neutrophil recruitment to the lung in $S d c 4^{-/-}$mice after bleomycin treatment (Figure 3, C and D). To determine whether neutrophil deficiency contributes to fibrogenesis in syndecan-4 deficiency, we used the neutrophil chemoattractant $N$-formylneoleucyl-leucyl-phenylalanine (FNLP) to forcibly recruit neutrophils into alveolar spaces (26). After intratracheal instillation of FNLP with bleomycin into $S d c 4^{-/-}$mice, neutrophil numbers were restored to levels comparable to those of WT mice (Supplemental Figure 2A). Interestingly, restoring neutrophils in the alveolar space did not alter the exaggerated fibrotic phenotype observed in $\mathrm{Sdc} 4^{-/}$ mice after noninfectious lung injury (Supplemental Figure 2B). 


\section{A}

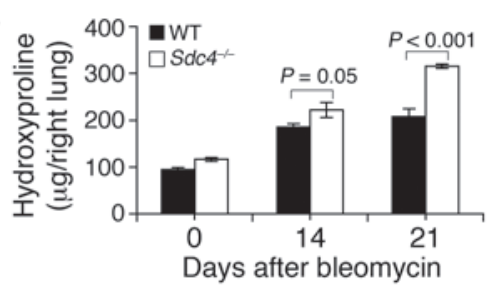

B WT

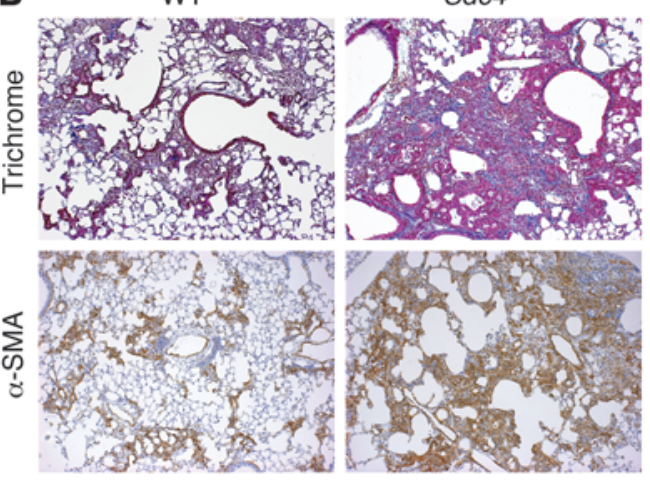

C
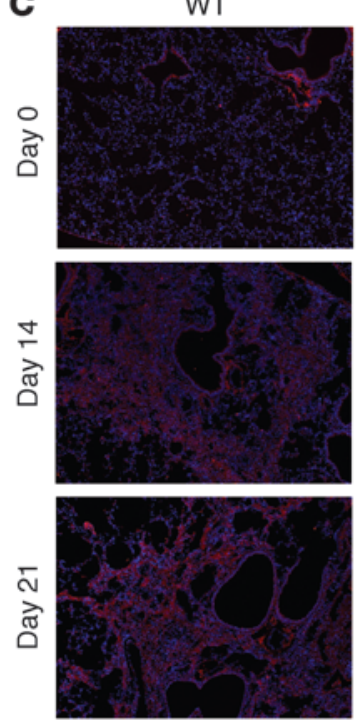

$S d c 4^{-\leftarrow}$
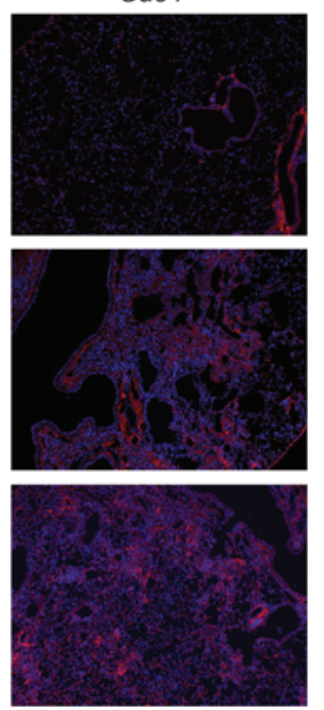

Figure 2

Increased pulmonary fibrosis in Sdc4 $4^{--}$mice. (A) Sdc4 $4^{--}$and littermate WT controls were subject to bleomycin-induced lung injury. Hydroxyproline contents in the lung were measured 14 and 21 days after injury $(n=5-10)$. Data are representative of 3 similar experiments. (B) Lung sections of Sdc4-/- and WT mice 21 days after injury were stained with trichrome and anti- $\alpha$-SMA, showing increased collagen staining and $\alpha$-SMA in Sdc4 $4^{--}$mice $(n=7)$. Original magnification, $\times 100$. (C) Lung sections of Sdc4 $4^{--}$and WT mice before and 14 and 21 days after injury were stained for collagen I, showing increased collagen I staining in lung sections of Sdc $4^{-/-}$mice $(n=7)$. Original magnification, $\times 100$.

These data suggest that although syndecan-4 contributes to neutrophil transepithelial migration, the exaggerated fibrogenesis in $S d c 4^{-/-}$mice is not caused by impaired neutrophil recruitment; other mechanisms must account for the fibrotic phenotype.

Inbibition of fibrosis by CXCL10 requires the presence of syndecan- 4 . An exhaustive search to identify an increase in profibrotic mediators, such as TGF- $\beta$ or IL-13, or a decrease in antifibrotic cytokines, such as IFN- $\gamma$ and HGF, failed to identify candidates to account for the marked increase in fibroblast accumulation in $\mathrm{Sdc} 4^{-/-}$mice (Supplemental Figure 3). The failure to find alterations in pro- or

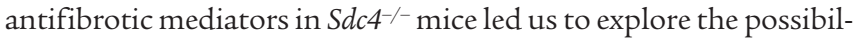
ity that syndecan- 4 might have a role in limiting lung fibroblast migration. Chemokines and chemokine receptors are known to regulate fibroblast migration. CXCR3 and CXCL10 have previously been shown to limit lung fibrosis in the bleomycin model (9-11). Because CXCL10 has been shown to interact with heparan sulfate (27), we sought to determine whether exogenous CXCL10 affects in vivo fibrogenesis in the absence of syndecan-4. Exogenous administration of CXCL10 inhibited bleomycin-induced lung fibrosis in WT mice (Figure 4), as previously reported (9). However, the antifibrotic effect of CXCL10 was lost in $\mathrm{Sdc4} 4^{-/-}$mice (Figure 4), demonstrating that the presence of syndecan-4 was required for this effect.

Increased accumulation of fibroblasts in $\mathrm{Sdc} 4^{-/-}$mouse lungs after bleomycin injury. Fibroblasts are the main effector cells during fibrogenesis. They accumulate in the lung interstitium after injury and produce matrix in response to critical mediators, such as TGF- $\beta$. Increased fibrosis in $S d c 4^{-/}$mice in bleomycin injury model may be caused by imbalances in fibroblast proliferation, collagen synthesis, or migration. We confirmed that CXCL10 did not affect fibroblast proliferation (data not shown), as previously reported (10). We then determined whether CXCL10 plays a role in regulating collagen synthesis. Mouse fibroblast NIH 3T3 cells were treated with a recombinant mouse CXCL10 protein. CXCL10 did not affect collagen production by $3 \mathrm{~T} 3$ fibroblasts (Supplemental Figure 4). Importantly, we confirmed that TGF- $\beta$ stimulated collagen production by fibroblasts and found that CXCL10 was unable to inhibit this effect. Thus, the antifibrotic effect of CXCL10 is not caused by collagen synthesis inhibition. However, lung sections stained with the fibroblast marker ER-TR7 (10) did reveal a substantial increase in fibroblast staining in $\mathrm{Sdc4} 4^{-/}$mouse lungs after bleomycin injury (Figure 5). These data suggest that a critical component of the fibrotic phenotype in $S d 4^{-/-}$mice is increased accumulation of fibroblasts at the site of tissue injury.

CXCL10 directly interacts with syndecan-4 on lung fibroblasts. CXCL10 was previously found to inhibit fibroblast chemotaxis in response to BAL fluid from injured lung tissue (10). The mechanism for this inhibition was unclear, since lung fibroblasts did not express the CXCL10 receptor CXCR3 (Supplemental Figure 5 and ref. 10). Because CXCL10 has previously been shown to bind glycosaminoglycans (GAGs; ref. 27), we hypothesized that CXCL10 might bind to syndecan-4 on fibroblasts in order to regulate fibroblast migration. We investigated the possibility that CXCL10 binds directly to syndecan-4 using several approaches. First, we found that the binding of iodine-labeled CXCL10 to lung single-cell homogenates or cultured primary fibroblasts of $S d c 4^{-/}$mice was reduced compared with homogenates from lung tissue of WT mice (Figure 6, A and B). Total lung cells isolated from $\mathrm{Cxcr}^{-/-}$mice also showed reduced binding of CXCL10 compared with homogenates from WT mouse lung tissue (Figure 6A). CXCL10 binding to $\mathrm{CxCr}^{-/-}$fibroblasts was 
A

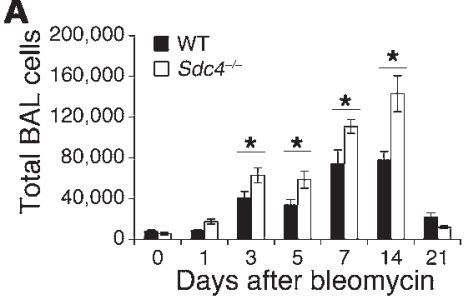

C

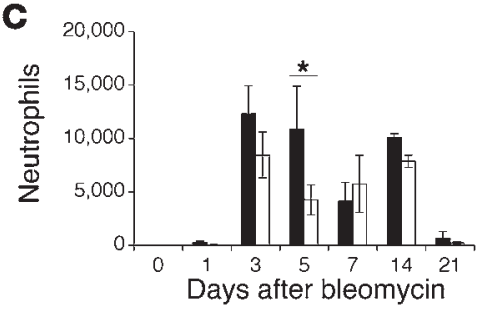

E

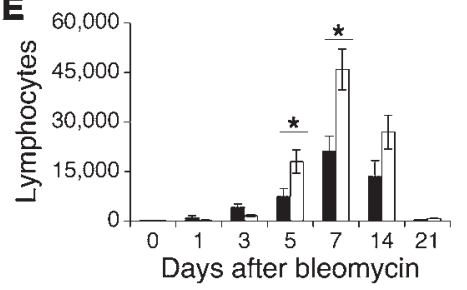

B

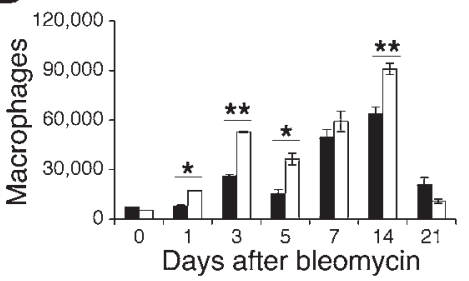

D

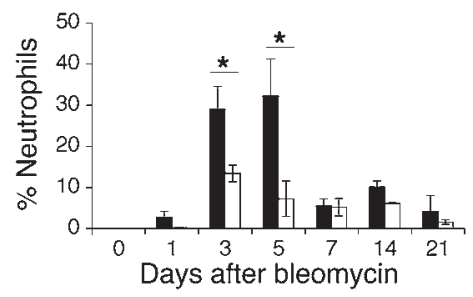

Figure 3

Dysregulated inflammation in Sdc4-/- mice after bleomycin lung injury. (A) Sdc4-- mice showed increased total inflammatory cells in BAL during a 21-day period after bleomycininduced lung injury. (B-E) The total cell increase was a result of an increase in macrophages (B) and lymphocytes (E). (C and D) There was also a decrease in neutrophils. (A-E) $n=5-7 .{ }^{*} P<0.05 ;{ }^{* \star} P<0.01$. Similar results were obtained in 3 separate experiments. comparable to that of WT fibroblasts (Figure 6B). This binding was specific, since the binding activity was blocked by the addition of unlabeled CXCL10 (Figure 6C). Similar results were obtained from total lung cells isolated from mice (data not shown). Second, biotinylated CXCL10 crosslinked to syndecan-4-bearing cells, and the binding was abolished by the addition of unlabeled CXCL10 protein (Figure 6D). As a control, CXCL10 was demonstrated to be crosslinked to CXCR3-bearing cells, and the crosslinking was displaced by the addition of unlabeled CXCL10 (data not shown). These data demonstrated that CXCL10 directly binds to syndecan- 4 .

CXCL10-syndecan-4 interactions regulate fibroblast migration. We then sought to determine the biological significance of CXCL10-syndecan- 4 interaction in the pathogenesis of pulmonary fibrosis by investigating its effects on fibroblast migration. BAL from patients with pulmonary fibrosis $(28,29)$ or from bleomycin-treated mice $(10,29)$ elicits chemotactic activity for fibroblasts. CXCL10 is able to inhibit BAL-induced fibroblast migration (10). To determine whether syndecan-4 is required for the CXCL10 inhibition of fibroblast chemotaxis, we examined the migration of both WT and $S d c 4^{-/-}$fibroblasts in response to BAL fluid from fibrotic lung tissue and observed migration in both groups (Figure 7, A and B). Consistent with our previous observation, recombinant CXCL10 inhibited the chemotaxis of primary lung fibroblasts from WT mice in response to BAL fluid isolated from the lungs of mice treated with bleomycin (Figure 7, A and B). However, CXCL10 did not attenuate $S d c 4^{-/}$fibroblast migration in response to BAL. Thus, the inhibition of fibroblast chemotaxis by CXCL10 is dependent on the presence of syndecan- 4 on fibroblasts. Furthermore, CXCR3 was dispensable for CXCL10 inhibition of fibroblast chemotaxis, since CXCL10 inhibited BAL-induced fibroblast migration from $\mathrm{CxCr}^{-/-}$fibroblasts (Figure 7C).

The heparin-binding domain, but not the CXCR3-binding domain, of CXCL10 is crucial for inhibition of fibroblast migration. The CXCL10 crystal structure was recently determined (30), and the structural domains of CXCL10 protein have been investigated by mutational studies $(31,32)$. The $\mathrm{N}$-terminal arginine residue of CXCL10 protein, preceding the first cysteine, is important for CXCR3 signaling, whereas mutations of the 4 basic residues of the $\mathrm{C}$-terminal helix (K71E/R72Q/K74Q/R75E) combined with the R22A mutation (CtR22A) abolish most of the heparin-binding activity of CXCL10 (32). We next determined whether inhibition of BAL-induced fibroblast migration by CXCL10 requires the heparin binding site, using 2 mutants of WT CXCL10 protein in fibroblast migration studies. Mutations were made in either the heparin-binding domain or the CXCR3-binding domain. An alanine exchange of residue Arg-8 of CXCL10 protein (R8A), which abrogates CXCR3 binding, did not change the ability of CXCL10 to inhibit BAL-induced fibroblast migration, whereas CtR22A, which abolishes heparin binding,

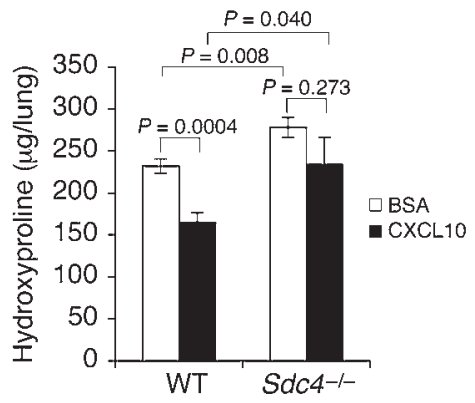

Figure 4

Exogenous CXCL10 did not inhibit fibrosis in Sdc4 $4^{-/-}$mice. Sdc4 $4^{-/-}$and littermate WT controls were subject to bleomycin-induced lung injury. Recombinant mouse CXCL10 or BSA buffer was given intramuscularly daily from the day of injury. Hydroxyproline contents in the lung were measured 13 days after injury $(n=8-9)$. 


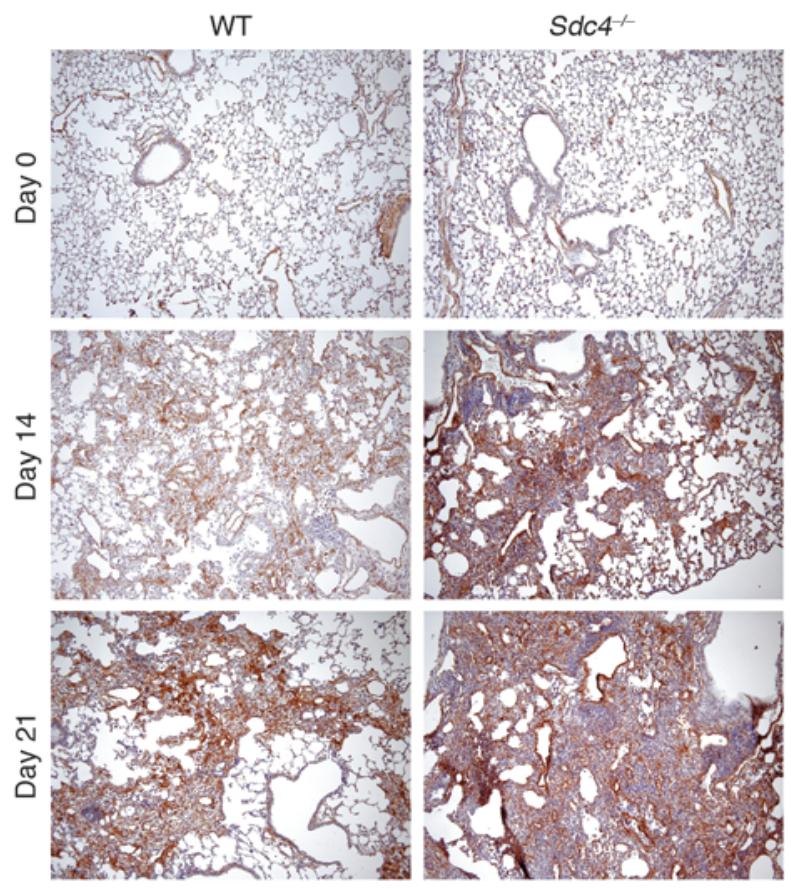

resulted in a significant loss of the inhibitory effect on fibroblast migration (Figure 8A). These observations suggest that the inhibitory effect of CXCL10 resides in its heparin-binding sites. Thus, the CXCL10-CXCR3 interaction is dispensable for inhibiting BALinduced fibroblast migration, whereas the GAG binding region of CXCL10 is critical. CXCL10 binding to cells can be inhibited by heparin and heparan sulfate (27). To further confirm the requirement of a CXCL10-GAG interaction in the inhibition of fibroblast migration, we examined the effect of GAG blockade using heparan sulfate and heparin. Heparan sulfate and heparin reduced the inhibition of CXCL10 on BAL-induced fibroblast migration in a dose-dependent manner (Figure 8B and data not shown). Furthermore, pretreatment of cultured primary fibroblasts with heparinase reduced the inhibition of CXCL10 on BAL-induced fibroblast migration (Figure 8C). Collectively, these data suggest that CXCL10 evokes its inhibitory effect on BAL-induced fibroblast migration through CXCL10-GAG interactions on the cell surface.

To determine the clinical relevance of CXCL10 inhibition on fibroblast migration, we tested whether CXCL10 protein inhibits migration of human lung fibroblasts. Syndecan-4 was expressed on human lung fibroblasts (data not shown). Interestingly, primary human fibroblasts isolated from patients with progressive idiopathic pulmonary fibrosis (IPF) migrated in response to BAL fluid isolated from bleomycin-treated mouse lungs. BAL-induced fibroblast migration was blocked by murine CXCL10 protein (Figure $8 \mathrm{D})$. We then determined that R8A inhibited BAL-induced fibroblast migration, similar to WT CXCL10 (Figure 8D). Taken together, these data suggest that CXCL10 directly interacts with syndecan-4 and that this interaction is required for CXCL10-mediated inhibition of fibroblast chemotaxis in vitro and the antifibrotic effect of CXCL10 in vivo.

\section{Discussion}

Syndecan-4 has been recognized as an important component in the formation of focal adhesion complexes through interactions with

\section{Figure 5}

More fibroblast staining in Sdc4 $4^{-/-}$versus WT mouse lungs. Lung sections of Sdc4- and littermate WT mice before and 14 and 21 days after bleomycin injury were stained with fibroblast marker ER-TR7, showing increased fibroblast staining in lung sections of $\mathrm{Sdc} 4^{-/}$mice $(n=7)$. Original magnification, $\times 100$.

integrins in vitro (12). Recently, $S d c 4^{-/-}$fibroblasts were found to migrate randomly as a result of high activity of delocalized Rac-1 (33). However, $S d c 4^{-/-}$mice develop normally, which suggests that these in vitro effects are not required for normal development. Syndecan- 4 has been shown to affect wound healing, resulting in delayed closure of skin incisions in its absence (20). Syndecan-4 is required for dermal fibroblast invasive migration into fibrin clot (34). We identified $S d c 4$ as a gene induced in response to lung injury and sought to determine its role in lung inflammation and fibrosis. Although we anticipated that there would be impaired fibrogenesis in the absence of syndecan-4, we unexpectedly found the opposite: a marked increase in collagen deposition relative to WT mice after noninfectious lung injury.

Several studies have suggested that CXCL10 and its cognate receptor are important in regulating the fibroproliferative response. Keane and colleagues first showed that exogenous CXCL10 inhibited fibrogenesis (9). Genetically targeting the deletion of the CXCL10 receptor CXCR3 resulted in increased mortality with progressive interstitial fibrosis after lung injury, demonstrating a nonredundant role for CXCR3 in limiting tissue fibroproliferation (11). Cxcl10 $0^{-/-}$mice also exhibited an enhanced fibrogenic response to bleomycin, and CXCL10 was found to inhibit fibroblast migration in response to BAL from injured lungs (10). However, since fibroblasts do not express CXCR3, the mechanism of inhibition must involve another cell surface receptor. Our current study demonstrates what we believe to be a previously unrecognized interaction between syndecan-4 and CXCL10 in the regulation of fibrogenesis. We provide 2 lines of direct biochemical evidence for an interaction between CXCL10 and syndecan-4. Iodine-labeled CXCL10 bound to syndecan-4-positive fibroblasts, and the binding was competed away with unlabeled CXCL10. In addition, biotinylated CXCL10 was detected in syndecan-4 immunoprecipitates.

In order to determine whether the CXCL10-syndecan-4 interaction was biologically relevant, we used a bioassay for lung fibroblast migration. It has been known for decades that after acute lung injury, chemoattractants for fibroblasts accumulate in the lung (6, 28). However, the precise components of the inflammatory milieu that account for this activity have never been completely elucidated. Candidates include growth factors, such as PDGF (35) and IGF-1 (36), and matrix components, such as fibronectin, collagen, and hyaluronan (37). It has recently been shown that lysophosphatidic acid may be the critical mediator of chemotactic activity for fibroblast migration (29). In the present study, we confirmed that the chemotaxis of primary lung fibroblasts to injured lung BAL fluid was inhibited by exogenous CXCL10 (10). However, the ability of CXCL10 to inhibit BAL-induced fibroblast migration was dependent on the presence of both syndecan- 4 and a functional GAG-binding domain in the CXCL10 protein. CXCL10 did not inhibit BAL-induced migration of $S d c 4^{-/-}$fibroblasts, which suggests that the CXCL10-syndecan-4 interaction is required for this activity. To determine whether CXCL10 affects other fibroblast 

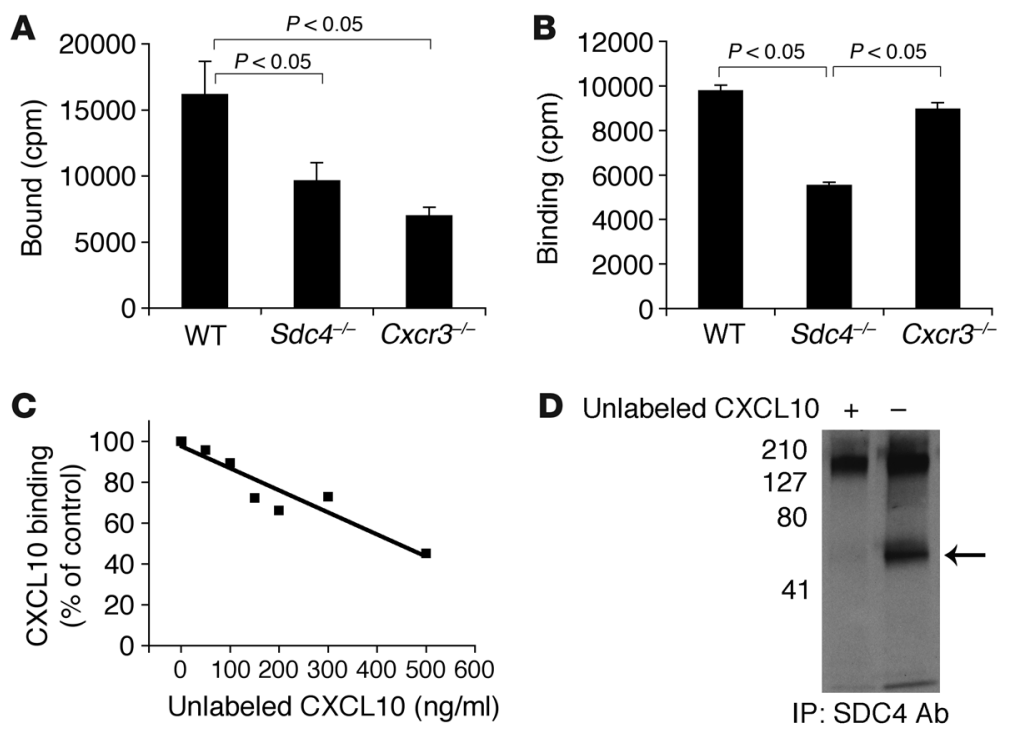

\section{Figure 6}

CXCL10 binds to syndecan-4. (A) Equal numbers of cells isolated from lung tissue of WT, Sdc4 $4^{-/}$, and $\mathrm{CxCr}^{-/-}$mice were incubated with ${ }^{125}$-labeled CXCL10. After wash, total radiolabel activity was measured. Decreased binding of ${ }^{125}$ |-labeled CXCL10 to Sdc4 $4^{--}$and $\mathrm{CXCr}^{-/-}$cells was observed $(n=3)$. Similar results were obtained from 3 experiments. (B) Equal numbers of fibroblasts isolated from lung tissue of WT, Sdc4 ${ }^{-1-}$, and $\mathrm{CXCr} 3^{-/-}$mice were incubated with ${ }^{125}$-labeled CXCL10. Decreased binding of ${ }^{125}$ I-labeled CXCL10 to Sdc $4^{-/-}$fibroblasts was observed $(n=4)$. Similar results were obtained from 3 experiments. (C) Binding of CXCL10 to fibroblasts. Syndecan-4-bearing fibroblasts from WT lung tissue were plated to confluence in 24-well plates and incubated with ${ }^{125}$-labeled CXCL10 and increasing concentrations of unlabeled CXCL10 at $4^{\circ} \mathrm{C}$. The amount of $125 \mathrm{I}-\mathrm{CXCL} 10$ bound to cells is shown as the percentage of that in the absence of unlabeled CXLC10. (D) Crosslinking CXCL10 to syndecan-4. Biotin-labeled CXCL10 $(0.5 \mu \mathrm{g})$ was incubated with lung tissue homogenates in the presence or absence of an excess amount of unlabeled CXCL10, crosslinked with DSS, and followed by IP with antibody specific to syndecan-4. Crosslinked syndecan-4 with biotinylated CXCL10 was visualized with streptavidin-HRP followed by enhanced chemiluminescence. Arrow denotes the CXCL10-syndecan-4 complex.

effector functions, we examined effects on proliferation and collagen synthesis. CXCL10 did not inhibit fibroblast proliferation or block TGF- $\beta$-stimulated collagen production. These data, coupled with the increased accumulation of fibroblasts observed in $S d c 4^{-/-}$ mice after injury, as documented by immunohistochemical staining, directly implicate fibroblast trafficking as the site of action for CXCL10-syndecan-4 interactions.

Syndecan-4 contains 3 Ser-Gly consensus motifs for heparan sulfate attachment sites (25). Through its heparan sulfate side chains, syndecan-4 interacts with an array of extracellular proteins, including growth factors such as bFGF-2 (14) and TGF- $\beta$ (15), chemokines (16), adhesion molecules (13), and other extracellular matrix proteins $(12,38)$. CXCL10-syndecan-4 interactions may also occur through the carbohydrate chain of syndecan-4. Mutation of the GAG-binding domain of CXCL10 abolished its inhibitory effect on BAL-induced fibroblast migration. Furthermore, disruption of potential CXCL10-syndecan-4 interactions through the GAGbinding domain with either heparin or heparan sulfate attenuated the inhibitory effect of CXCL10 protein on BAL-induced fibroblast migration. In addition, removal of GAG from the cell surface by heparinase significantly reduced CXCL10 inhibition of BAL-induced fibroblast chemotaxis. Syndecan-4 is upregulated during tissue inflammation and injury (39), and we have now shown it to be upregulated on lung fibroblasts. A recent study suggested that fibroblast responses to fibronectin fragments are mediated by a fibrin-integrin-tenascin C-syndecan-4 complex (40). One possibility is that CXCL10 prevents fibronectin from interacting with syndecan-4.

In addition to the regulation of fibroblast migration, there are several additional potential mechanisms, such as increased epithelial apoptosis $(41,42)$, Th1/Th2 imbalance $(10,11,43)$, epithelial-to-mesenchymal transition $(44,45)$, increased fibrocyte recruitment (23), and altered stem/progenitor cell functions (46-48), which could also account for the enhanced fibrosis in $S d c 4^{-/-}$mice after bleomycin treatment. Matrix protein CCN1 (CYR61) interacts with syndecan-4 and integrins regulating prostate cancer cell apoptosis (49). Alveolar type II cell apoptosis has been suggested as an important early feature in the pathogenesis of pulmonary fibrosis (50-52). TGF- $\beta$ has been shown to play a central role in fibrosis $(53,54)$. We did not detect any differences in TGF- $\beta$ levels in Sdc4-/- and WT mouse lung tissues in the bleomycin model; more sensitive assays may be needed to detect TGF- $\beta$ under these conditions. However, a recent study showed that prostaglandin Fprostaglandin F receptor signaling mediates pulmonary fibrosis independently of TGF- $\beta$ in the bleomycin lung injury model (55). Syndecan-4 and other proteins have been suggested to comprise a population of myogenic precursor cells that differentiate and fuse with myotubes and, when transplanted, regenerate and maintain a substantial, functional population of muscle progenitor cells (56). Further investigations are needed to dissect other mechanisms by which syndecan-4 regulates fibrogenesis.

CXCL10 is upregulated after both immune-mediated and non-immune-mediated tissue injury (11, 57-59). The effect of CXCL10 on T cell recruitment is dependent on CXCR3 $(20,58)$. CXCL10-CXCR3 interactions have been directly implicated in the pathogenesis of immune-mediated tissue damage $(58,60-62)$. We and others have shown that after sterile lung injury, CXCL10 is produced and has antifibrotic properties (9-11). However, unlike immune-mediated pathobiology, we found that the antifibrotic effects of CXCL10 were independent of CXCR3 and required GAG binding and the presence of the heparan sulfated proteoglycan syndecan-4. We further demonstrated that the CXCL10 mutant R8A, which is deficient in CXCR3 binding, inhibited human fibroblast migration. These data suggest that a mutant CXCL10, without CXCR3-binding activity, delivered directly to the lungs could be a novel therapeutic agent in pulmonary fibrosis. Eliminating CXCR3 binding could avoid potential toxicities related to $\mathrm{T}$ cell activation. We have shown that syndecan-4 plays an important role in the host response to noninfectious lung injury, that syndecan- 4 is required for the antifibrotic effects of CXCL10 in vivo, and that the ability of CXCL10 to inhibit fibroblast migration in vitro occurs independent of the CXCR3 receptor and requires the GAG-binding domain of syndecan-4. Exploiting the interactions between proteoglycans and chemokines could lead to new therapeutic options in progressive pulmonary fibrosis. 
A

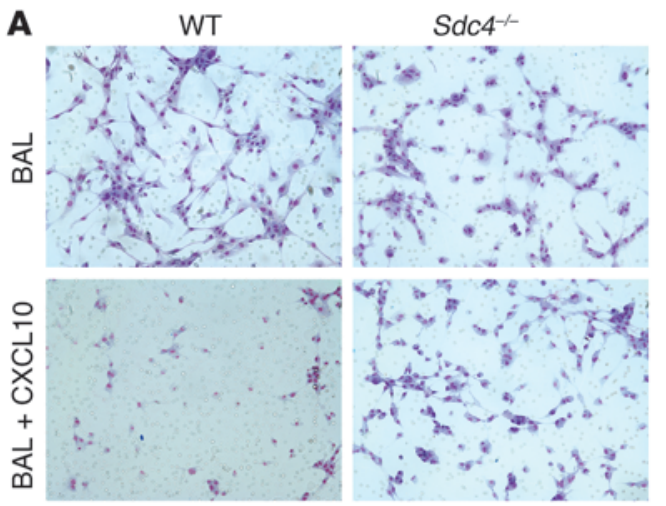

B
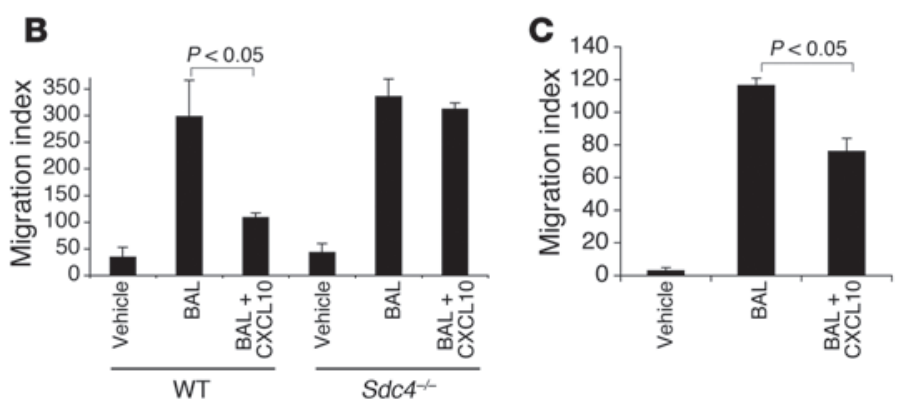

Figure 7

CXCL10 inhibition of BAL-induced fibroblast chemotaxis requires syndecan-4. (A) Lung fibroblasts were isolated from Sdc4-/- and littermate control mice and plated onto fibronectin-coated Boyden chambers with a pore size of $8 \mu \mathrm{m}$. BAL from WT mice 5 days after bleomycin injury was added to the bottom chambers. For certain groups, recombinant CXCL10 $(500 \mathrm{pg} / \mathrm{ml})$ was also added to the top and bottom chambers $(n=12-14)$. Original magnification, $\times 100$. Similar results were obtained from 5 experiments. (B) Quantitation of fibroblast migration. Fibroblasts were counted in 5 fields per slide under light microscopy with $\mathrm{a} \times 40$ objective lens. The total number of fibroblasts in 5 fields was expressed as a migration index $(n=3-5)$. (C) Lung fibroblasts were isolated from $\mathrm{CxCr}^{-1-}$ mice and plated onto Boyden chambers. BAL from WT mice 5 days after bleomycin injury was added to the bottom chambers. For certain groups, recombinant CXCL10 was also added to the top and bottom chambers. The total number of fibroblasts in 4 fields under microscope with $a \times 40$ objective lens was expressed as migration index $(n=4)$.

\section{Methods}

Mice. Sdc4 $4^{-/-}$mice were described previously (20). Mice were backcrossed onto C57BL/ 6 background for more than 6 generations before use. Ageand sex-matched background C57BL/6J mice were from Jackson. $\mathrm{Cxcr3} 3^{-/-}$ mice were provided by C. Gerard (Harvard Medical School, Boston, Massachusetts, USA). All mice were housed and cared for in a pathogen-free facility at Duke University, and all animal experiments were approved by the Institutional Animal Care and Use Committee at Duke University.

Bleomycin administration and BAL. Bleomycin was administered to $S d c 4^{-1}$ and age- and sex-matched WT C57BL/6J mice as described previously (11). Under anesthesia, the mouse lungs and heart were surgically exposed. The trachea was cannulated, and the lungs were lavaged 3 times with $0.8-\mathrm{ml}$ aliquots of cold PBS. The live cells were recovered and counted using a hemocytometer. Cytospin preparations of BAL cells were stained conventionally, and differential cell counts were performed.

Exogenous CXCL10 treatment. Both $S d c 4^{-/}$mice and littermate controls were divided into CXCL10 treatment and control groups, with 10 mice per group. All $S d \mathrm{c}^{-/-}$mice and littermate controls were subject to bleomycin-induced lung injury. Recombinant mouse CXCL10 (1 $\mu \mathrm{g}$; Peprotech) dissolved in $20 \mu \mathrm{l}$ of $0.25 \% \mathrm{BSA} / \mathrm{PBS}$ was given to mice intramuscularly daily from the day of injury. As a control, $20 \mu \mathrm{l}$ of $0.25 \% \mathrm{BSA} / \mathrm{PBS}$ was given to a separate group of mice intramuscularly every day. The mouse

\section{Figure 8}

A critical role for the GAG-binding site of CXCL10 protein in inhibition of BAL-induced fibroblast chemotaxis. Lung fibroblasts were isolated from WT mice $(\mathbf{A}-\mathbf{C})$ or from pulmonary fibrosis patients (D), plated onto Boyden chambers, and treated as detailed below. Fibroblast migration was quantified by microscopy with $a \times 40$ objective lens, and fibroblasts were counted in 4 fields per slide ( $n=4$ per group). Data are expressed as migration index. (A and B) BAL from WT mice 5 days after bleomycin injury was added to the bottom chambers; for certain groups, recombinant native CXCL10, CXCL10 mutants R8A and CtR22A, or heparan sulfate (HS) was also added to the top and bottom chambers. (C) Some fibroblasts were treated with heparinase for 2 hours before migration assay. BAL was added to the bottom chambers, and $\mathrm{CXCL} 10$ was added to the top and bottom chambers. (D) Lung fibroblasts isolated from pulmonary fibrosis patients were treated with CXCL10, R8A, or CtR22A. Similar results were obtained from lung fibroblasts isolated from 3 different IPF patients.
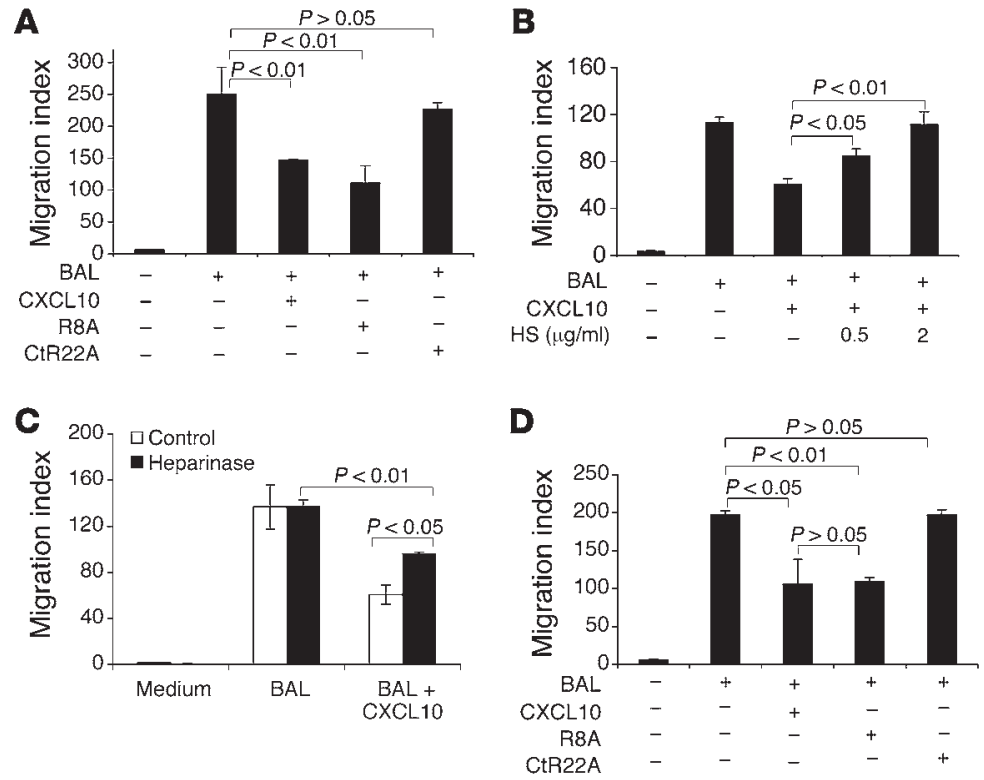
lungs were harvested 13 days after injury, and hydroxyproline contents in the lung were measured.

$m R N A$ analysis. RNA was extracted from lung tissues using TRIzOL reagent (Invitrogen) following the manufacturer's instructions. For realtime RT-PCR analysis, $1 \mu \mathrm{g}$ total RNA was reverse transcribed using SuperScript II RNase $\mathrm{H}^{-}$Reverse Transcriptase (Invitrogen) with $0.5 \mu \mathrm{g} / \mu \mathrm{l}$ oligo $(\mathrm{dT})_{17}$ primers. Of the resultant cDNAs, $1 \mu \mathrm{l}$ was subjected to real-time PCR (qPCR Core kit for SYBR Green; Eurogentec) using the ABI Prism 7500 Detection system (Applied Biosystems). The specific primers were designed based on cDNA sequences deposited in the GenBank database. Primers for Sdc4 (NM_011521) were 5'-ACTGGATAACCACATCCCTGAG-3' (sense) and 5'-GCAACTGAGACACAGACACACA-3' (antisense), and primers for GAPDH (NM_00100130) were 5'-ATCATCTCCGCCCCTTCTG-3' (sense) and 5'-GGTCATGAGCCCTTCCACAAT-3' (antisense). The primers were all cDNA specific, not amplifying genomic DNA. Contamination of genomic DNA was negligible. The conditions for real-time PCR were as follows: 1 cycle at $95^{\circ} \mathrm{C}$ for 10 minutes, 40 cycles at $95^{\circ} \mathrm{C}$ for 15 seconds and $60^{\circ} \mathrm{C}$ for 1 minute, and 1 cycle at $25^{\circ} \mathrm{C}$ for 2 minutes. The relative expression levels of each gene were determined against GAPDH levels in the samples.

Flow cytometry. Flow cytometric analysis on whole lung single-cell homogenates or isolated primary lung fibroblasts was carried out as described previously (11). Cell surface expression of syndecan-4 was detected with a phycoerythrin-conjugated specific antibody to sydnecan-4 (clone KY/8.2; BD Biosciences), along with leukocyte surface marker CD45 on a flow cytometer (FACSCanto II; Becton Dickson). Cell surface expression of syndecan-4 was also analyzed with a sydnecan-4 antibody (provided by P. Kincade, Oklahoma Medical Research Foundation, Oklahoma City, Oklahoma, USA). Flow data were analyzed with FACS Diva software (Becton Dickson).

Histology and immunohistochemistry. Collagen contents were detected by conventional Trichrome staining of lung sections, as described previously (11). $\alpha$-SMA in lung tissue was stained with anti- $\alpha$-SMA (clone 1A4; DakoCytomation), collagen I was stained with a rabbit polyclonal antibody to collagen I (Abcam), and fibroblasts were stained with ER-TR7 (Santa Cruz Biotechnology) and followed by visualization with a Vectastain ABC kit (Vector Laboratories) or fluorescently.

Hydroxyproline assay. Collagen contents in mouse lungs were measured with a conventional hydroxyproline method (11).

Radiolabeled CXCL10 binding. Cells of lung single-cell homogenates or purified lung fibroblasts $\left(1 \times 10^{5}\right)$ were incubated with ${ }^{125}$ I-CXCL10 (PerkinElmer) and various concentrations of unlabeled CXCL10 (R\&D Systems) for 60 minutes at $4{ }^{\circ} \mathrm{C}$. The cells were filtered through glass microfiber filters (Whatman) treated with $0.33 \%$ polyethylenimine, washed, air dried, and counted on a gamma counter.

Crosslinking. Recombinant CXCL10 was from R\&D Systems. CXCL10 was biotinylated using EZ-Link Sulfosuccinimidyl-6'-(biotinamido)-6-hexanamido hexanoate (Pierce Biotechnology). Protein from lung homogenates
$(1 \mathrm{mg})$ was incubated with $0.5 \mu \mathrm{g}$ biotinylated CXCL10 at $4^{\circ} \mathrm{C}$ overnight; some of the samples were preincubated with $1 \mu \mathrm{g}$ CXCL10 at room temperature for 1 hour. Crosslinking was performed with reagent DSS on ice for 2 hours. IP was done with anti-syndecan-4 antibody (clone KY103; ref. 63) or CXCR3 antibody (Zymed). Immunoprecipitates were separated on a $4 \%-20 \%$ Tris-SDS polyacrylamide gel electrophoresis, transferred onto PVDF membrane, and visualized with enhanced chemiluminescence (Amersham).

Isolation of primary lung fibroblasts and fibroblast migration assay. Primary lung fibroblasts were isolated and cultured, and the fibroblast chemotaxis assay was performed as described previously (10). Lung fibroblasts were plated onto Boyden chambers with pore size of $8 \mu \mathrm{m}$ coated with fibronectin (10). BAL from WT mice 3 days after bleomycin injury was added to the bottom chambers. Certain groups were also treated with recombinant CXCL10 or CXCL10 mutants, at a concentration of 500-1000 ng/ $\mathrm{ml}$, or in combination with heparin or heparan sulfate at $0.5-2 \mu \mathrm{g} / \mathrm{ml}$, added to the top and bottom chambers. Heparinase I from Flavobacterium heparinum was from Sigma-Aldrich. Fibroblasts $\left(1 \times 10^{6}\right.$ cells $)$ were treated with heparinase at $0.02 \mathrm{U}$ for 2 hours (64). The migration index was expressed as total number of cells in 5 fields examined with $\times 100$ or $\times 400$ magnification under microscope.

Human lung fibroblasts. Human lung fibroblasts were isolated from surgical lung biopsies from patients with IPF. The diagnosis of IPF was arrived at by standard accepted American Thoracic Society recommendations (65). All experiments were approved by the Duke University Institutional Review Board and were in accordance with the guidelines outlined by the board. Informed consent was obtained from each subject.

Statistics. Differences in measured variables between genetically altered mice and the control group or between treatment groups were assessed using Student's 2-tailed $t$ test using JMP software (SAS Institute). Data are expressed as mean \pm SEM where applicable. A $P$ value less than 0.05 was considered a statistically significant difference.

\section{Acknowledgments}

We thank P. Kincade for providing anti-syndecan-4 antibody and C. Gerard for providing $\mathrm{C} x \mathrm{Cr} 3^{-/-}$mice. This study was supported by NIH grants AI052201, HL06539, and HL77291 to P.W. Noble.

Received for publication January 20, 2009, and accepted in revised form March 24, 2010.

Address correspondence to: Paul W. Noble or Dianhua Jiang, Division of Pulmonary, Allergy, and Critical Care Medicine, Department of Medicine, Duke University School of Medicine, 106 Research Drive, Durham, North Carolina 27710, USA. Phone: 919.681.0355; Fax: 919.684.5266; E-mail: paul.noble@duke.edu (P.W. Noble); dianhua.jiang@duke.edu (D. Jiang).
1. Thannickal VJ, Toews GB, White ES, Lynch JP 3rd, Martinez FJ. Mechanisms of pulmonary fibrosis. Annu Rev Med. 2004;55:395-417.

2. Noble PW, Homer RJ. Idiopathic pulmonary fibrosis: new insights into pathogenesis. Clin Chest Med.2004; 25(4):749-758, vii.

3. Kuwano K, et al. Essential roles of the Fas-Fas ligand pathway in the development of pulmonary fibrosis. J Clin Invest. 1999;104(1):13-19.

4. Ramos C, et al. Fibroblasts from idiopathic pulmonary fibrosis and normal lungs differ in growth rate, apoptosis, and tissue inhibitor of metalloproteinases expression. Am J Respir Cell Mol Biol. 2001; 24(5):591-598.

5. Selman M, et al. TIMP-1, $-2,-3$, and -4 in idiopathic pulmonary fibrosis. A prevailing nondegradative lung microenvironment? Am J Physiol Lung Cell Mol
Physiol. 2000;279(3):L562-L574.

6. Suganuma H, Sato A, Tamura R, Chida K. Enhanced migration of fibroblasts derived from lungs with fibrotic lesions. Thorax. 1995;50(9):984-989.

7. Bonner JC. Regulation of PDGF and its receptors in fibrotic diseases. Cytokine Growth Factor Rev. 2004; 15(4):255-273.

8. Belperio JA, et al. CXC chemokines in angiogenesis. J Leukoc Biol. 2000;68(1):1-8.

9. Keane MP, et al. IFN-gamma-inducible protein-10 attenuates bleomycin-induced pulmonary fibrosis via inhibition of angiogenesis. J Immunol. 1999; 163(10):5686-5692.

10. Tager AM, et al. Inhibition of Pulmonary Fibrosis by the Chemokine IP-10/CXCL10. Am J Respir Cell Mol Biol. 2004;31(4):395-404.

11. Jiang D, et al. Regulation of pulmonary fibrosis by chemokine receptor CXCR3. J Clin Invest. 2004; 114(2):291-299.

12. Couchman JR. Syndecans: proteoglycan regulators of cell-surface microdomains? Nat Rev Mol Cell Biol. 2003;4(12):926-937.

13. Saoncella S, et al. Syndecan-4 signals cooperatively with integrins in a Rho-dependent manner in the assembly of focal adhesions and actin stress fibers. Proc Natl Acad Sci U S A. 1999;96(6):2805-2810.

14. Horowitz A, Tkachenko E, Simons M. Fibroblast growth factor-specific modulation of cellular response by syndecan-4. J Cell Biol. 2002;157(4):715-725.

15. Ishiguro K, Kojima T, Muramatsu T. Syndecan-4 as a molecule involved in defense mechanisms. GlycoconjJ. 2002;19(4-5):315-318.

16. Hamon $M$, et al. A syndecan-4/CXCR4 complex expressed on human primary lymphocytes and 
macrophages and HeLa cell line binds the CXC chemokine stromal cell-derived factor-1 (SDF-1). Glycobiology. 2004;14(4):311-323.

17. Woods A, Couchman JR. Syndecan-4 and focal adhesion function. Curr Opin Cell Biol. 2001; 13(5):578-583.

18. Ishiguro K, et al. Syndecan-4 deficiency increases susceptibility to kappa-carrageenan-induced renal damage. Lab Invest. 2001;81(4):509-516

19. Ishiguro K, et al. Syndecan-4 deficiency leads to high mortality of lipopolysaccharide-injected mice. J Biol Chem. 2001;276(50):47483-47488.

20. Echtermeyer F, et al. Delayed wound repair and impaired angiogenesis in mice lacking syndecan-4. J Clin Invest. 2001;107(2):R9-R14.

21. Evans MJ, et al. Atypical development of the tracheal basement membrane zone of infant rhesus monkeys exposed to ozone and allergen. Am J Physiol Lung Cell Mol Physiol. 2003;285(4):L931-L939.

22. Dull RO, et al. Lung endothelial heparan sulfates mediate cationic peptide-induced barrier dysfunction: a new role for the glycocalyx. Am J Physiol Lung Cell Mol Physiol. 2003;285(5):L986-L995.

23. Phillips RJ, et al. Circulating fibrocytes traffic to the lungs in response to CXCL12 and mediate fibrosis. J Clin Invest. 2004;114(3):438-446.

24. Smith RE, et al. Production and function of murine macrophage inflammatory protein-1 alpha in bleomycin-induced lung injury. J Immunol. 1994; 153(10):4704-4712.

25. Zhang K, Rekhter MD, Gordon D, Phan SH. Myofibroblasts and their role in lung collagen gene expression during pulmonary fibrosis. A combined immunohistochemical and in situ hybridization study. Am J Pathol. 1994;145(1):114-125.

26. Li Q, Park PW, Wilson CL, Parks WC. Matrilysin shedding of syndecan-1 regulates chemokine mobilization and transepithelial efflux of neutrophils in acute lung injury. Cell. 2002;111(5):635-646.

27. Luster AD, Greenberg SM, Leder P. The IP-10 chemokine binds to a specific cell surface heparan sulfate site shared with platelet factor 4 and inhibits endothelial cell proliferation. J Exp Med. 1995;182(1):219-231.

28. Behr J, Adelmann-Grill BC, Krombach F, Beinert T, Schwaiblmair M, Fruhmann G. Fibroblast chemotactic response elicited by native bronchoalveolar lavage fluid from patients with fibrosing alveolitis. Thorax. 1993;48(7):736-742.

29. Tager AM, et al. The lysophosphatidic acid receptor LPA1 links pulmonary fibrosis to lung injury by mediating fibroblast recruitment and vascular leak. Nat Med. 2008;14(1):45-54.

30. Swaminathan GJ, et al. Crystal structures of oligomeric forms of the IP-10/CXCL10 chemokine. Structure. 2003;11(5):521-532.

31. Campanella GS, et al. Oligomerization of CXCL10 is necessary for endothelial cell presentation and in vivo activity. J Immunol. 2006;177(10):6991-6998.

32. Campanella GS, Lee EM, Sun J, Luster AD. CXCR3 and heparin binding sites of the chemokine IP-10 (CXCL10). J Biol Chem. 2003;278(19):17066-17074.
33. Bass MD, et al. Syndecan-4-dependent Rac1 regulation determines directional migration in response to the extracellular matrix. J Cell Biol. 2007; 177(3):527-538.

34. Lin F, Ren XD, Doris G, Clark RA. Three-dimensional migration of human adult dermal fibroblasts from collagen lattices into fibrin/fibronectin gels requires syndecan-4 proteoglycan. J Invest Dermatol. 2005;124(5):906-913.

35. Antoniades $\mathrm{HN}$, et al. Platelet-derived growth factor in idiopathic pulmonary fibrosis. J Clin Invest. 1990;86(4):1055-1064.

36. Uh ST, Inoue Y, King TE Jr, Chan ED, Newman LS, Riches DW. Morphometric analysis of insulinlike growth factor-I localization in lung tissues of patients with idiopathic pulmonary fibrosis. Am J Respir Crit Care Med. 1998;158(5 pt 1):1626-1635.

37. Bensadoun ES, Burke AK, Hogg JC, Roberts CR. Proteoglycan deposition in pulmonary fibrosis. Am J Respir Crit Care Med. 1996;154(6 pt 1):1819-1828.

38 . Kon S, et al. Syndecan-4 protects against osteopontin-mediated acute hepatic injury by masking functional domains of osteopontin. J Exp Med. 2008; 205(1):25-33.

39. Kojima T, et al. Plasma levels of syndecan-4 (ryudocan) are elevated in patients with acute myocardial infarction. Thromb Haemost. 2001;85(5):793-799.

40. Midwood KS, Valenick LV, Hsia HC, Schwarzbauer JE. Coregulation of fibronectin signaling and matrix contraction by tenascin- $C$ and syndecan- 4 . Mol Biol Cell. 2004;15(12):5670-5677.

41. Martin TR, Hagimoto N, Nakamura M, MatuteBello G. Apoptosis and epithelial injury in the lungs. Proc Am Thorac Soc. 2005;2(3):214-220.

42. Henson PM, Vandivier RW, Douglas IS. Cell death, remodeling, and repair in chronic obstructive pulmonary disease? Proc Am Thorac Soc. 2006;3(8):713-717.

43. Jakubzick C, et al. Therapeutic attenuation of pulmonary fibrosis via targeting of IL-4- and IL-13-responsive cells. J Immunol. 2003;171(5):2684-2693.

44. Willis BC, et al. Induction of epithelial-mesenchymal transition in alveolar epithelial cells by transforming growth factor-beta1: potential role in idiopathic pulmonary fibrosis. Am J Pathol. 2005;166(5):1321-1332.

45. Kim KK, et al. Alveolar epithelial cell mesenchymal transition develops in vivo during pulmonary fibrosis and is regulated by the extracellular matrix. Proc Natl Acad Sci U S A. 2006;103(35):13180-13185.

46. Hashimoto N, Jin H, Liu T, Chensue SW, Phan SH. Bone marrow-derived progenitor cells in pulmonary fibrosis. J Clin Invest. 2004;113(2):243-252.

47. Gomperts BN, Strieter RM. Stem cells and chronic lung disease. Annu Rev Med. 2007;58:285-298.

48. Chua F, Gauldie J, Laurent GJ. Pulmonary fibrosis: searching for model answers. Am J Respir Cell Mol Biol. 2005;33(1):9-13

49. Franzen CA, Chen CC, Todorovic V, Juric V, Monzon RI, Lau LF. Matrix protein CCN1 is critical for prostate carcinoma cell proliferation and TRAIL-induced apoptosis. Mol Cancer Res. 2009;7(7):1045-1055.

50. Noble PW. Idiopathic pulmonary fibrosis: natu- ral history and prognosis. Clin Chest Med. 2006; 27(1 suppl 1):S11-S16, v.

51. Noble PW, Homer RJ. Back to the future: historical perspective on the pathogenesis of idiopathic pulmonary fibrosis. Am J Respir Cell Mol Biol. 2005;33(2):113-120.

52. Selman M, Pardo A. Role of epithelial cells in idiopathic pulmonary fibrosis: from innocent targets to serial killers. Proc Am Thorac Soc. 2006;3(4):364-372.

53. Khalil N, Greenberg AH. The role of TGF-beta in pulmonary fibrosis. Ciba Found Symp. 1991; 157:194-207; discussion 207-211.

54. Gauldie J, Kolb M, Ask K, Martin G, Bonniaud P, Warburton D. Smad3 signaling involved in pulmonary fibrosis and emphysema. Proc Am Thorac Soc. 2006; 3(8):696-702

55. Oga T, et al. Prostaglandin F(2alpha) receptor signaling facilitates bleomycin-induced pulmonary fibrosis independently of transforming growth factor-beta. Nat Med. 2009;15(12):1426-1430.

56. Tanaka KK, Hall JK, Troy AA, Cornelison DD, Majka SM, Olwin BB. Syndecan-4-expressing muscle progenitor cells in the SP engraft as satellite cells during muscle regeneration. Cell Stem Cell. 2009; 4(3):217-225

57. Zhai Y, et al. CXCL10 regulates liver innate immune response against ischemia and reperfusion injury. Hepatology. 2008;47(1):207-214.

58. Hancock WW, Gao W, Csizmadia V, Faia KL, Shemmeri N, Luster AD. Donor-derived IP-10 initiates development of acute allograft rejection. J Exp Med. 2001;193(8):975-980.

59. Dixon AE, Mandac JB, Madtes DK, Martin PJ, Clark JG. Chemokine expression in Th1 cell-induced lung injury: prominence of IFN-gamma-inducible chemokines. Am J Physiol Lung Cell Mol Physiol. 2000;279(3):L592-L599.

60. Belperio JA, et al. Critical role for CXCR3 chemokine biology in the pathogenesis of bronchiolitis obliterans syndrome. J Immunol. 2002;169(2):1037-1049.

61. Medoff BD, et al. CXCR3 and its ligands in a murine model of obliterative bronchiolitis: regulation and function. J Immunol. 2006;176(11):7087-7095.

62. Hancock WW, et al. Requirement of the chemokine receptor CXCR3 for acute allograft rejection. J Exp Med. 2000;192(10):1515-1520.

63. Yamashita Y, Oritani K, Miyoshi EK, Wall R, Bernfield M, Kincade PW. Syndecan-4 is expressed by B lineage lymphocytes and can transmit a signal for formation of dendritic processes. J Immunol. 1999; 162(10):5940-5948

64. Richardson TP, Trinkaus-Randall V, Nugent MA. Regulation of basic fibroblast growth factor binding and activity by cell density and heparan sulfate. J Biol Chem. 1999;274(19):13534-13540.

65. ATS/ERS. American Thoracic Society. Idiopathic pulmonary fibrosis: diagnosis and treatment. International consensus statement. American Thoracic Society (ATS), and the European Respiratory Society (ERS). Am J Respir Crit Care Med. 2000; 161(2 pt 1):646-664. 